\title{
Inappropriate shocks delivered by implantable cardiac defibrillators during oversensing of activity of diaphagmatic muscle
}

\author{
D Babuty, L Fauchier, P Cosnay
}

\begin{abstract}
Two cases are reported (both men, one 72 and one 54 years old) of inappropriate shocks delivered by an implantable cardiac defibrillator (ICD) device, which oversensed the myopotentials induced by deep breathing and Valsalva manoeuvre. No damage to leads was associated with the oversensing of myopotentials. The mechanism of the inappropriate shocks was determined using real time electrograms. Modification of the duration of ventricular detection and decrease in sensitivity made it possible to avoid the oversensing of myopotentials and to deliver ICD treatment.
\end{abstract} (Heart 1999;81:94-96)

Keywords: implantable cardiac defibrillator; inappropriate shocks; myopotentials

Delivery of inappropriate shocks by implantable cardioverter defibrillators (ICDs) is a common complication. ${ }^{12}$ The mostfrequent cause is the occurrence of supraventricular tachycardia that cannot be differentiated by the ICD from ventricular tachycardia. ${ }^{12}$ Other causes are oversensing of $\mathrm{T}$ waves, pacing artefacts from a separate pacemaker, and noise from electronic devices, and lead damage. ${ }^{1-6}$ We report, for the first time, inappropriate shock by oversensing of diaphragmatic muscular activity in two patients implanted with third and fourth generation ICDs.

\section{Case 1}

A 74 year old man with coronary heart disease and recurrent ventricular fibrillation refractory to amiodarone and $\beta$ blocker treatment received a pectoral ICD (Mini1763; CPI Inc, St Paul, Minnesota, USA) with a bipolar endocardial catheter (Endotak model 0125; CPI Inc) in the right ventricular apex. The intraoperatively sensed $\mathrm{R}$ wave amplitude was measured at $8 \mathrm{mV}$ and the pacing threshold was $0.7 \mathrm{~V}$ at $0.5 \mathrm{~ms}$ pulse width. The ICD was programmed
Accepted for publication 24 June 1998
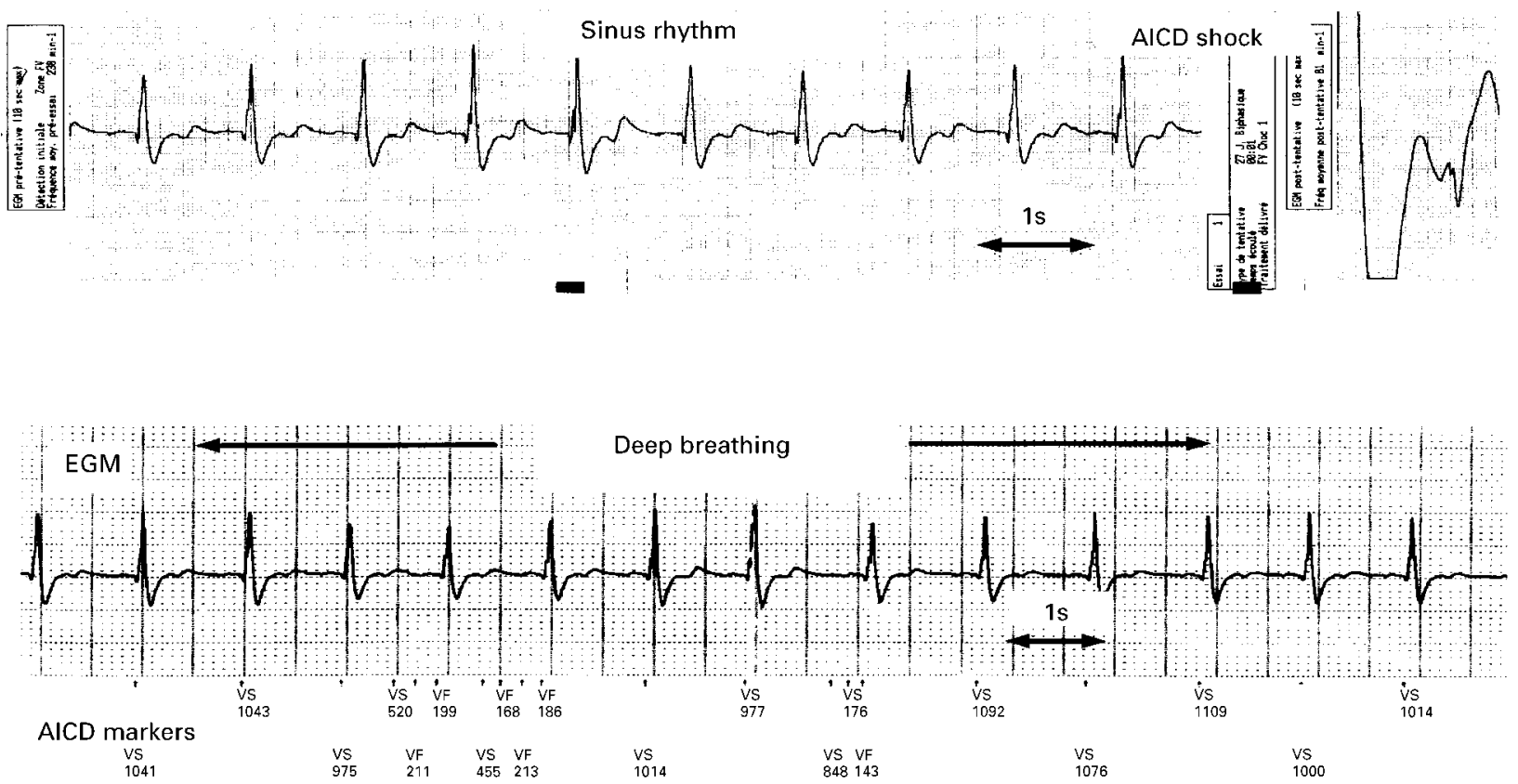

Figure 1 Recording of real time ECG (top) and ECG during deep breathing (bottom) from case 1 showing oversensing of myopotentials, identified as ventricular fibrillation (VF) by the ICD (AICD markers). 


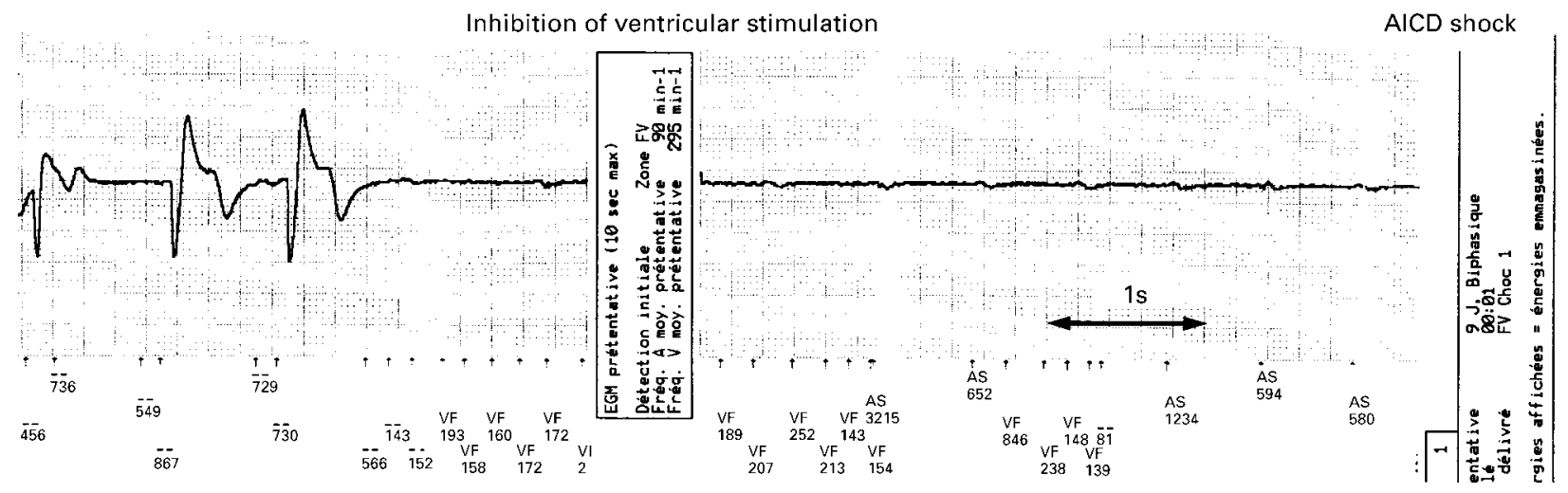

Figure 2 Restored ECG from case 2 showing sinus rhythm with inhibition of ventricular stimulation by myopotentials and activation of the ICD.

to a detection interval of $316 \mathrm{~ms}$ during $1 \mathrm{sec}-$ ond of ventricular fibrillation. The detection algorithm was initially 8 of 10 then 6 of 10 consecutive RR intervals for ventricular tachycardia. Five days later the patient received two shocks during defecation stress without dizziness or syncope. Interrogation of the device revealed detection of ventricular fibrillation with a mean RR interval of $238 \mathrm{~ms}$ whereas the stored ECG showed a sinus rhythm at 65 beats/min (fig 1). The lead impedance was not changed ( $585 \mathrm{Ohms}$ ) during checking of the device, nor were the pacing threshold $(0.6 \mathrm{~V}$ at $0.5 \mathrm{~ms})$ or the sensing lead ( $\mathrm{R}>5 \mathrm{mV}$ ). Chest radiography did not show fracture or dislodgement of the lead.

To determine the mechanism of electrical signal oversensing, we recorded the ECG with real time intracardiac electrograms during deep breathing and Valsalva manoeuvre. Figure 1 shows an example of ECG recording simultaneous sinus rhythm and the detection of ventricular fibrillation. To avoid inappropriate shock, we programmed a longer duration of detection in the ventricular fibrillation zone ( 2 seconds). No relapse was observed during nine months of follow up with the newly programmed parameters.

\section{Case 2}

A 54 year old man with right ventricular arrhythmogenic dysplasia and recurrence of monomorphic ventricular tachycardia underwent radiofrequency catheter ablation of the emergency site. At the end of the procedure, clinical ventricular tachycardia was no longer inducible but faster ventricular tachycardia was induced. Ablation of the second emergency site was attempted but complete and permanent
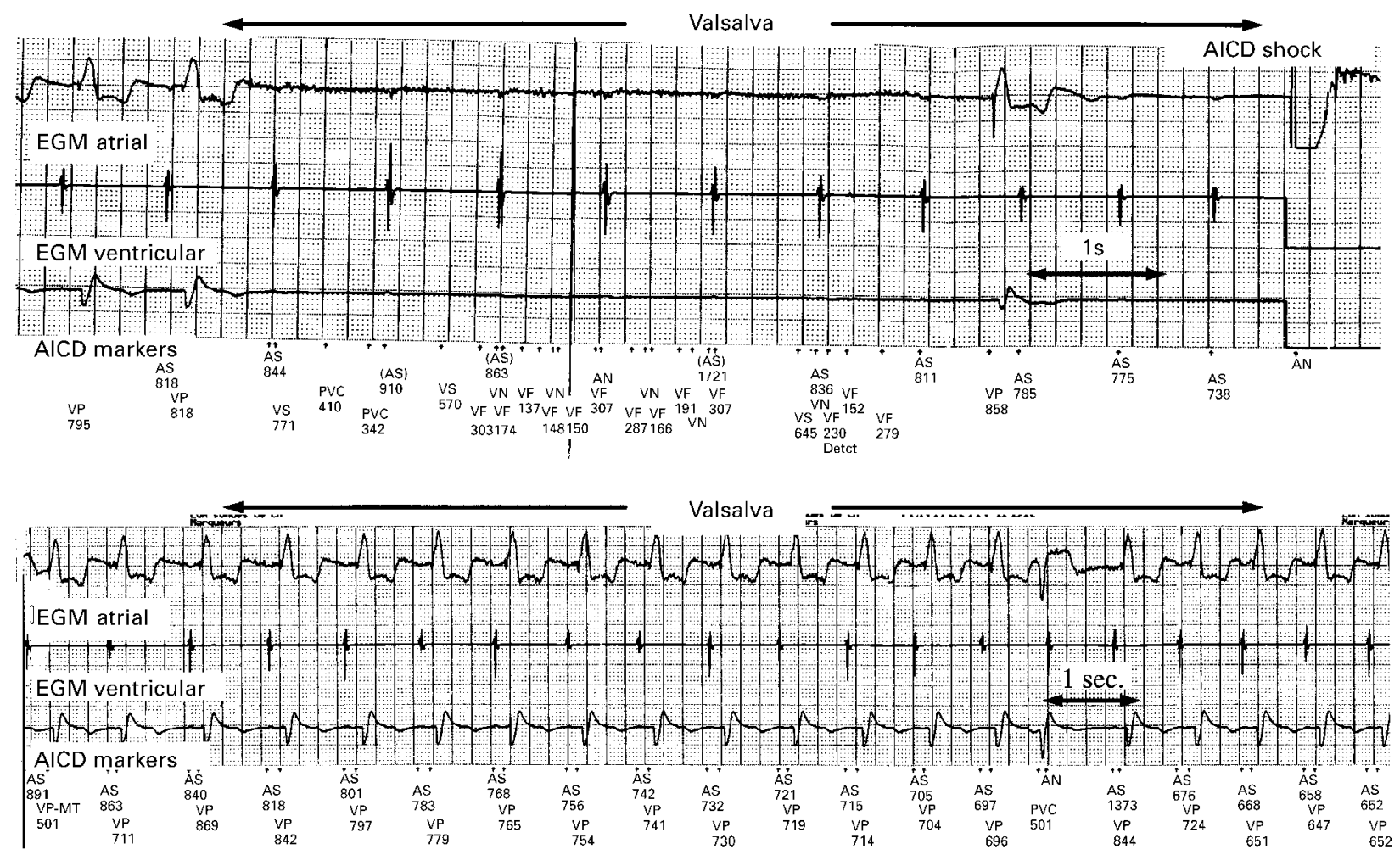

Figure 3 (Top) ECG and real time intracardiac electrograms obtained during the Valsalva manoeuvre in case 2 showing multiple events that were identified as rapid spontaneous ventricular activity (VF) resulting in inhibition of ventricular pacing and activation of the ICD. (Bottom) After changing the sensitivity of the device the same manoeuvre was not associated with myopotential sensing. 
atrioventricular block occurred, justifying implantation of an ICD. The patient received a pectoral ICD (AV II DR 1821; CPI Inc) with one ventricular bipolar endocardial catheter (Endotak model 0125; CPI Inc) and one auricular bipolar lead (Sweet Tip Rx model 4244; CPI Inc). The ICD was programmed to a detection interval of $333 \mathrm{~ms}$ during 1 second of ventricular fibrillation. Permanent antibradycardia function was activated (pacing mode DDD, minimal and maximal frequencies from 60 to 120 beats/min). The sensitivity of detection was nominal. Two days after surgery, the patient complained of syncope in the sitting position during a Valsalva manoeuvre and received one shock. Interrogation of the device showed the detection of ventricular fibrillation whereas the stored ECG showed complete atrioventricular block followed by one shock delivered by the ICD device (fig 2). After having eliminated dysfunction of the lead, we reproduced the electrical phenomenon by the Valsalva manoeuvre: myopotentials inhibited ventricular stimulation and were identified as ventricular fibrillation by the ICD device. As shown in fig 3, we programmed lower sensitivity, which permitted suppression of the phenomenon.

\section{Discussion}

Inappropriate shocks are a common complication in patients with an ICD device. ${ }^{12}$ The most frequent cause is the occurrence of rapid supraventricular tachycardia. ${ }^{12}$ In the absence of damage to leads, electrical interference with ICD devices has rarely been identified as a cause of inappropriate shocks. ${ }^{356}$ For the first time, we report inappropriate shocks delivered by recently implanted ICD devices in two patients, secondary to sensed myopotentials during deep breathing or the Valsalva manoeuvre. There was no lead dysfunction previously reported in either case. ${ }^{147}$ No change in lead impedance was measured and no fracture or dislodgement was observed on chest radiographs. The conditions of occurrence were the same in both patients: a few days after implantation of the device, in a sitting position, during deep breathing, and during the Valsalva manoeuvre. In both cases there was extensive dilatation of the right ventricle and the defibrillator leads were positioned at the apex of the right ventricle, near the diaphragm.
Moreover by recording real time ECGs it was possible to demonstrate that the mechanism was an oversensing of the activity of the respiratory muscles. The problem was solved by changing the sensitivity of the device. However, in the third generation device (case 1) this parameter is not accessible to the physician and we prolonged the time of detection of ventricular fibrillation to avoid inappropriate shock. In the second patient, oversensing of myopotentials had two consequences: syncopal atrioventricular block and subsequent inappropriate shock. The small amplitude of the muscular potentials was detected by the ICD device, which automatically increased the sensitivity to prevent undersensing of ventricular fibrillation. This automatic gain algorithm cannot be suppressed but only modulated in these devices. Three levels of sensitivity are possible: nominal, moderate, and minimal. Programming at the minimal level in case 2 was the solution.

The limit of the automatic gain algorithm was reported by Curwin et al who described a case of inappropriate shock by oversensing of atrial activity with another model of ICD device (Cadence model V-100; Ventritex Sylman, California, USA) ${ }^{8}$ For the future, it is important that the sensitivity of the ICD device is programmable, as with antibradycardia devices, if this type of complication is to be avoided.

1 Pinski SL, Fahy GJ. The proarrythmic potential of implantable cardioverter-defibrillators. Circulation 1995;92:165164.

2 Nunain SO, Roelke M, Trouton T, et al. Limitations and late complications of third-generation automatic cardioverterdefibrillators. Circulation 1995;91:2204-13.

3 Man KC, Davidson T, Langberg JJ, et al. Interference from hand radiofrequency remote control causing discharge of an implantable defibrillator. PACE 1993;16:1756-8.

4 Sandler MJ, Kutalek SP. Inappropriate discharge by an implantable cardioverter defibrillator: recognition of myopotential sensing using telemetered intracardiac electrograms. PACE 1994;17:665-71.

5 Seifert T, Block M, Borgreggrefe $M$, et al. Erroneous discharge of an implantable cardioverter defibrillator caused by an electric razor. PACE 1995;19:1592-4

6 Mathew P, Lewis C, Neglia J, et al. Interaction between electronic article surveillance systems and implantable defibrillators: insights from a fourth generation ICD. $P A C E$ 1997;20:2857-9.

7 Hief C, Podczeck A, Frohner K, et al. Cardioverter discharges following sensing of electrical artifact due to discharges following sensing of electrical artifact due to 1589-91.

8 Curwin JA, Roelke M, Ruskin JN. Inhibition of bradycardia pacing caused by far-field atrial sensing in a thirdgeneration cardioverter defibrillator with an automatic gain feature. PACE 1996;19:124-6. 